\title{
Comparing Experimental and Calculated Electron-Capture Rates of pf-shell Nuclei in Explosive Stellar Environments
}

\author{
A.L. Cole ${ }^{*}{ }^{\dagger a}$ T.S. Anderson, ${ }^{a}$ Sam M. Austin, ${ }^{b c d}$ B.A. Brown, ${ }^{b c d}$ A.C. Dombos, ${ }^{a}$ O. \\ Fawwaz, ${ }^{e}$ S. Gupta, ${ }^{f}$ G.W. Hitt, ${ }^{e}$ A.K. Schooley, ${ }^{a}$ L. Valdez, ${ }^{b c d}$ R.G.T. Zegers ${ }^{b c d}$ \\ ${ }^{a}$ Physics Department, Kalamazoo College, Kalamazoo, MI 49006, USA \\ ${ }^{b}$ National Superconducting Cyclotron Laboratory, Michigan State University, East Lansing, MI \\ 48824, USA \\ ${ }^{c}$ Department of Physics and Astronomy, Michigan State University, East Lansing, MI 48824, USA \\ ${ }^{d}$ Joint Institute for Nuclear Astrophysics, Michigan State University, East Lasing, Michigan \\ 48824, USA \\ ${ }^{e}$ Department of Applied Mathematics and Sciences, Khalifa University of Science, Technology \\ and Research, P.O. Box 127788 Abu Dhabi, United Arab Emirates \\ ${ }^{f}$ Indian Institute of Technology Ropar, India
}

\begin{abstract}
In explosive stellar environments electron-capture rates on pf-shell nuclei are needed to model core-collapse and thermonuclear supernovae. Electron-capture (EC) rates can be determined from Gamow-Teller (GT) transitions in the $\beta^{+}$direction with strength $\mathrm{B}\left(\mathrm{GT}^{+}\right)$. These distributions can be extracted from charge-exchange measurements and from distributions calculated with theoretical models. In a recent study of 13 pf-shell nuclei with measured $\mathrm{B}\left(\mathrm{GT}^{+}\right)$distributions, we presented a systematic comparison between the electron-capture rates determined from measurements and those determined from shell model (with KB3G and GXPF1a interaction Hamiltonians) and quasiparticle random phase approximation (QRPA) calculations of $\mathrm{B}\left(\mathrm{GT}^{+}\right)$ distributions. The electron-capture rates derived from calculations were compared to rates derived from measurements at two stellar density (temperature) conditions, $\rho \mathrm{Y}_{e}=10^{7} \mathrm{~g} / \mathrm{cm}^{3}\left(3 \times 10^{9}\right.$ $\mathrm{K})$ and $\rho \mathrm{Y}_{e}=10^{9} \mathrm{~g} / \mathrm{cm}^{3}\left(10 \times 10^{9} \mathrm{~K}\right)$. In this work we summarize the results of the study.
\end{abstract}

XII International Symposium on Nuclei in the Cosmos,

August 5-12, 2012

Cairns, Australia

\footnotetext{
* Speaker.

$\dagger$ E-mail: acole@kzoo.edu
} 


\section{Introduction}

The dynamics of a core-collapse or thermonuclear supernova is influenced by the electroncapture rates on pf-shell nuclei $[1,2,3,4]$. Charge-exchange measurements and $\beta$-decay measurements can provide a means to experimentally determine electron-capture rates. As it's not feasible to measure the $\mathrm{B}\left(\mathrm{GT}^{+}\right)$distributions for all pf-shell nuclei, theoretical model calculations are needed for most nuclei and therefore must be tested. A recent paper, Ref. [5], presented results of a systematic comparison between the electron-capture rates determined from measured $\mathrm{B}\left(\mathrm{GT}^{+}\right)$distributions and the electron-capture rates determined from shell model and QRPA calculations of $\mathrm{B}\left(\mathrm{GT}^{+}\right)$distributions for the following 13 pf-shell nuclei: ${ }^{45} \mathrm{Sc},{ }^{48} \mathrm{Ti},{ }^{50} \mathrm{~V},{ }^{51} \mathrm{~V},{ }^{55} \mathrm{Mn}$, ${ }^{54} \mathrm{Fe},{ }^{56} \mathrm{Fe},{ }^{59} \mathrm{Co},{ }^{58} \mathrm{Ni},{ }^{60} \mathrm{Ni},{ }^{62} \mathrm{Ni},{ }^{64} \mathrm{Ni},{ }^{64} \mathrm{Zn}$. These nuclei were selected because there were existing charge-exchange measurements of the $\mathrm{B}\left(\mathrm{GT}^{+}\right)$distributions for each nucleus. The chargeexchange data sets considered included $11(\mathrm{n}, \mathrm{p})$ measurements, $6\left(\mathrm{~d},{ }^{2} \mathrm{He}\right)$ measurements and 2 $\left(\mathrm{t},{ }^{3} \mathrm{He}\right)$ measurements. The averages of the excitation-energy resolution of the $\mathrm{B}\left(\mathrm{GT}^{+}\right)$measurements were approximately $1.1 \mathrm{MeV}, 120 \mathrm{keV}$ and $260 \mathrm{keV}$ for the three reactions, respectively [5]. These charge-exchange measurements were augmented with $\beta^{-}$-decay measurements, which for four nuclei were used to determine the ground state strength. Additionally, for two nuclei, isospin symmetry was used to extract strength from $(p, n)$ measurements. For details of these 25 existing data sets, we refer the reader to Ref. [5] and the references therein.

We compared the measured GT distributions to theoretical distributions calculated with the QRPA and shell model (two interaction Hamiltonians) formalisms. The GT distributions are briefly described in Section 2. Electron-capture rates were determined from both the measured and calculated GT distributions at two stellar densities (temperatures). Section 3 briefly describes these calculations and compares the EC rates. Finally, in Section 4 we evaluate the deviations of the theoretical rates from those determined from experimental measurements and in Section 5 conclusions are presented.

\section{Gamow-Teller Strength Distribution Calculations}

We performed the calculations for Gamow-Teller transitions $\Delta \mathrm{S}=1, \Delta \mathrm{L}=0, \Delta \mathrm{T}_{z}=+1$ corresponding to the $\beta^{+}$and electron capture direction. Both of the shell model calculations were performed in the full model space. Furthermore, the model space was restricted to the pf-shell only and we only considered Gamow-Teller transitions from the ground state of the parent nuclei. The shell model calculations were performed with NUSHELLX@MSU $[6,7]$ using the pf-shell interaction Hamiltonians GXPF1a [8, 9, 10] and KB3G [11]. To facilitate comparison with the experimental measurements, the shell model calculations were quenched by the factor $(0.74)^{2}[12]$. The QRPA calculations followed the method of [13] with inputs from [14]. The QRPA results did not require quenching [13].

\section{Electron-Capture Rates Calculations}

The electron-capture rate calculations were performed with the code of Ref. [15] following the method of Fuller, Fowler and Newman (FFN) [16, 17, 18, 19]. The electron-capture rates 
were calculated at two stellar densities, $\rho \mathrm{Y}_{e}=10^{7} \mathrm{~g} / \mathrm{cm}^{3}$ and $\rho \mathrm{Y}_{e}=10^{9} \mathrm{~g} / \mathrm{cm}^{3}$ for a temperature range of $2-10 \times 10^{9} \mathrm{~K}$. Within these stellar environments, the electrons are in a Fermi-Dirac (FD) distribution with $\varepsilon_{F}=U_{F}+m_{e} c^{2}$, where $U_{F}$ is the electron chemical potential. The stellar density influences the electron chemical potential and, at a given density, the stellar temperature influences the degeneracy of the electrons. The electron-capture rate depends on the Gamow-Teller state excitation energy $\left(E_{e x}\right)$ and strength, the electron-capture Q-value $\left(Q_{E C}\right)$ and hence also depends on the reaction Q-value $\left(Q_{R}=E_{e x}-Q_{E C}\right)$. An electron capture occurs for $\varepsilon_{F}>Q_{R}$ (see Ref. [5] and references therein). At the lower density, the electrons first capture into the low energy B(GT) states. The rate increases strongly with temperature as the degeneracy decreases and more electrons gain enough energy to populate the states. At the higher stellar density the FD electron chemical potential is larger and hence electrons can populate more of the GT states. Electron-capture rates at the higher density have a weaker dependency on the temperature and location of the low energy states, depending more on the total GT strength [20,21]. Thus, by comparison, the electron-capture rates are larger at the higher density than at the lower density. See Ref. [5] for a detailed description along with figures of the electron-capture rates for each nucleus.

For the 13 nuclei considered, we calculated the electron-capture rate for at least four cases, rates determined from KB3G, GXPF1a and QRPA calculations and electron-capture rates determined from at least one charge-exchange measurement. To facilitate a comparison among these rates for different nuclei, we determined the ratios of the electron-capture rates $\left(\lambda_{E C}\right)$ to the GXPF1a rate $\left(\lambda_{E C}(\mathrm{GXPF1a})\right)$ at $\rho \mathrm{Y}_{e}=10^{7} \mathrm{~g} / \mathrm{cm}^{3}\left(3 \times 10^{9} \mathrm{~K}\right)$ and $\rho \mathrm{Y}_{e}=10^{9} \mathrm{~g} / \mathrm{cm}^{3}\left(10 \times 10^{9} \mathrm{~K}\right)$. These temperatures and densities approximately correspond to the conditions of Si-burning [2] and much later in the evolution before core collapse [4, 22], respectively (see Ref. [5] and the references therein). The ratio $\lambda_{E C}(\mathrm{~KB} 3 \mathrm{G}) / \lambda_{E C}(\mathrm{GXPF} 1 \mathrm{a})$ of the $\mathrm{KB} 3 \mathrm{G}$ electron capture rate to the GXPF1a electron capture rate at $\rho \mathrm{Y}_{e}=10^{7} \mathrm{~g} / \mathrm{cm}^{3}\left(3 \times 10^{9} \mathrm{~K}\right)$ varies by less than a factor of 10 and by less than a factor of 5 at $\rho \mathrm{Y}_{e}=10^{9} \mathrm{~g} / \mathrm{cm}^{3}\left(10 \times 10^{9} \mathrm{~K}\right)$ for the $13 \mathrm{pf}$-shell nuclei considered in Ref. [5]. Hence, the two shell model calculations show good agreement with each other at both densities (temperatures).

At the lower density (temperature), the $\lambda_{E C}(\mathrm{n}, \mathrm{p})$ to $\lambda_{E C}(\mathrm{GXPF} 1 \mathrm{a})$ ratio was greater than one for 10 of the 11 nuclei in the data set, exceeding a factor of 100 in two cases (See Figure 1(a)). The average of the excitation energy resolutions for the 11 (n,p) $\mathrm{B}\left(\mathrm{GT}^{+}\right)$measurements was approximately $1.1 \mathrm{MeV}$. This poor resolution results in some strength being assigned to artificially low excitation energies. Especially at relatively low stellar densities, this leads to a strong overestimate of the deduced electron-capture rates. The electron-capture rates determined from the $\left(\mathrm{d},{ }^{2} \mathrm{He}\right)$ and $\left(\mathrm{t},{ }^{3} \mathrm{He}\right)$ charge-exchange measurements showed better agreement with the $\lambda_{E C}(\mathrm{GXPF} 1 \mathrm{a})$, varying by less than a factor of 10. Finally, as shown in Figure 1, the QRPA electron-capture rates showed good agreement with the GXPF1a rate for some nuclei but differed by more than a factor of 100 for other nuclei. As the QRPA $\mathrm{B}\left(\mathrm{GT}^{+}\right)$distributions exhibited more strength and/or strength concentrated in fewer states than either of the shell model or experimental distributions, the agreement between the GXPF1a and QRPA electron-capture rates is not necessarily an indication of similarity between their respective Gamow-Teller strength distributions (see Ref. [5]). 

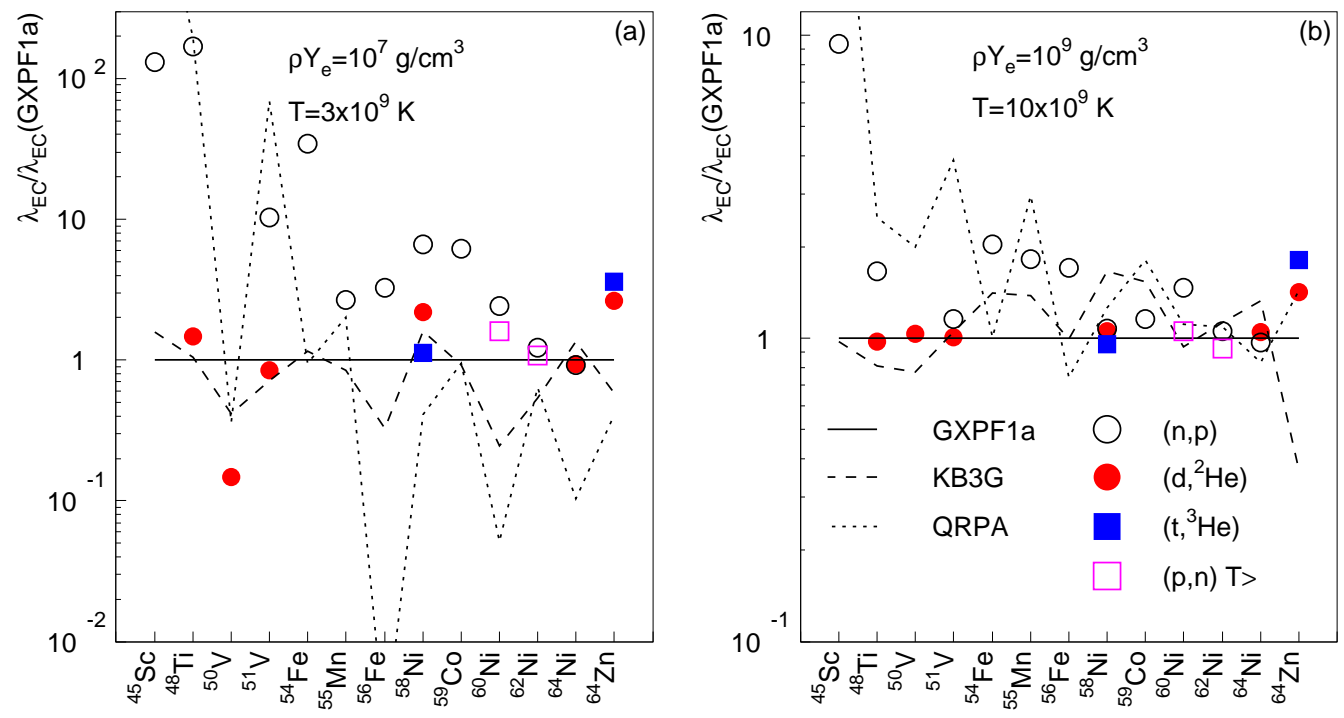

Figure 1: The ratio of the electron-capture rates $\left(\lambda_{E C}\right)$ determined from the measured and theoretical $\mathrm{B}\left(\mathrm{GT}^{+}\right)$distributions to the EC rate $\left(\lambda_{E C}(\mathrm{GXPF1a})\right)$ determined from the GXPF1a distribution are displayed for each of the 13 nuclei at a stellar density (temperature) of (a) $\rho \mathrm{Y}_{e}=10^{7} \mathrm{~g} / \mathrm{cm}^{3}\left(3 \times 10^{9} \mathrm{~K}\right)$ and (b) $\rho \mathrm{Y}_{e}=10^{9} \mathrm{~g} / \mathrm{cm}^{3}\left(10 \times 10^{9} \mathrm{~K}\right)$. (Data from Ref. [5].)

\section{Discussion}

To test the model calculations against the experimental measurements, we calculated the average deviation of the theoretically determined electron-capture rates $\left(\lambda^{\text {th }}\right)$ from the rates determined from measurements $\left(\lambda^{\exp }\right)[5]$ :

$$
\overline{\Delta_{E C}}=\frac{1}{N} \sum_{i=1}^{N} \frac{\lambda_{i}^{t h}-\lambda_{i}^{\exp }}{\lambda_{i}^{\exp }}
$$

The average deviation calculations only included nuclei with $\mathrm{B}\left(\mathrm{GT}^{+}\right)$distributions determined from high resolution $\left(\mathrm{d},{ }^{2} \mathrm{He}\right)$ and $\left(\mathrm{t},{ }^{3} \mathrm{He}\right)$ charge-exchange measurements or nuclei with well determined low energy Gamow-Teller states (See Ref. [5]). The EC rates derived from the (n,p) charge-exchange measurements were excluded as the relatively poor excitation energy resolution (see Section 3) measurements resulted in an artifical overestimation of the (n,p) EC rates for some nuclei. The results of the average deviation calculations are displayed in Figure 2(a) for seven nuclei, ${ }^{48} \mathrm{Ti},{ }^{51} \mathrm{~V},{ }^{58} \mathrm{Ni},{ }^{60} \mathrm{Ni},{ }^{62} \mathrm{Ni},{ }^{64} \mathrm{Ni}$ and ${ }^{64} \mathrm{Zn}$. For these same seven nuclei, we also calculated the average absolute deviations (Figure 2(b)) to ensure that differences between the theoretical and experimental rates were not obscured by the averaging of positive and negative deviations $\overline{\Delta_{E C}}[5]$ :

$$
\left|\overline{\Delta_{E C}}\right|=\frac{1}{N} \sum_{i=1}^{N} \frac{\left|\lambda_{i}^{t h}-\lambda_{i}^{\exp }\right|}{\lambda_{i}^{\exp }} .
$$

As displayed in Figure 2(a), the GXPF1a and KB3G electron-capture rates deviated less from the experimentally determined rates than the QRPA EC rates. At the lower density (temperature) the deviations were less than $40 \%$ for the shell model rates compared to a factor of 29 for the 

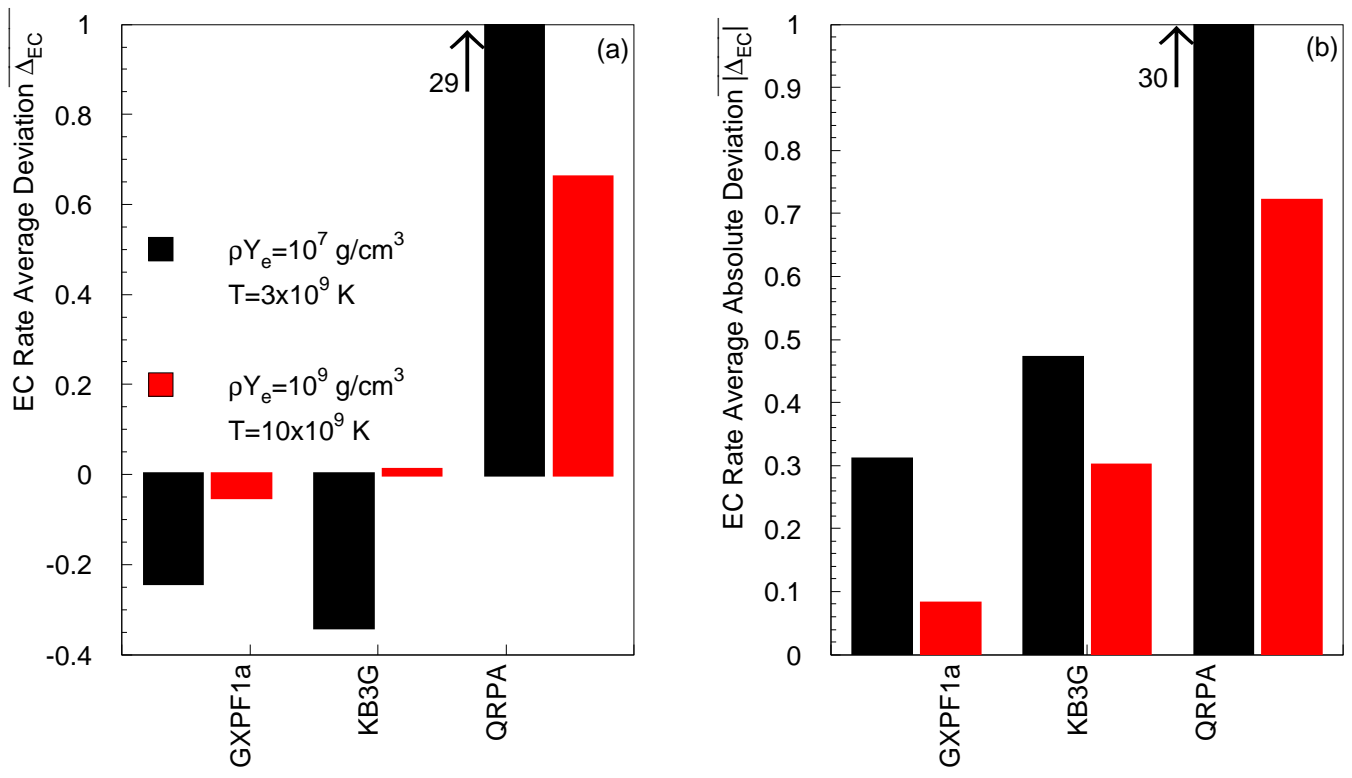

Figure 2: The (a) electron-capture rate average deviation $\left(\overline{\Delta_{E C}}\right)$ and the (b) electron-capture rate average absolute deviation $\left(\left|\overline{\Delta_{E C}}\right|\right)$ are displayed for the seven nuclei ${ }^{48} \mathrm{Ti},{ }^{51} \mathrm{~V},{ }^{58} \mathrm{Ni},{ }^{60} \mathrm{Ni},{ }^{62} \mathrm{Ni},{ }^{64} \mathrm{Ni}$ and ${ }^{64} \mathrm{Zn}$. The measured $\mathrm{B}\left(\mathrm{GT}^{+}\right)$distributions of these nuclei were determined from high resolution data or from well determined low energy GT states. (Data from Ref. [5], see reference for a detailed description.)

QRPA rates. While the deviations decreased, as expected, at the higher density, the average QRPA deviations were still considerably larger than those for the shell model calculations.

As displayed in Figure 2(b), the average absolute deviation of the electron-capture rates determined from the shell model $\mathrm{B}\left(\mathrm{GT}^{+}\right)$calculations differed by less than $50 \%$ at the lower density (temperature) and by $30 \%$ or less at the higher density (temperature). The QRPA average absolute deviation was large (a factor of 30) at the lower density (temperature) and, again, smaller at the higher density (temperature). However the QRPA deviations were still greater than the deviations for either shell model calculation.

\section{Conclusions}

We performed a systematic comparison of experimental and theoretical Gamow-Teller strength distributions for 13 nuclei in the pf-shell. Additionally, we compared ground-state EC rates deduced from these strength distributions. It was found that rates based on the strengths determined from the shell model calculations with the GXPF1a and KB3G interactions do much better in reproducing the rates determined from GT strengths of high resolution experiments than the rates determined from the QRPA calculations. Hence the KB3G and GXPF1a determined electron-capture rates are preferred as inputs for models of explosive stellar environments.

To further test the theoretical model calculations, we have begun to expand this initial study to include the calculation of electron-capture rates for pf-shell nuclei for which measurements do not exist and to consider the effect of excited states of the parent nuclei on the electron-capture rates. 
This work is supported in part by NSF grants PHY-0822648, PHY-0606007, PHY-0758099, PHY-1102511 and by an award from Research Corporation for Science Advancement.

\section{References}

[1] Bethe, H. A., Brown, G. E., Applegate, J., and Lattimer, J. M. (1979) Nucl. Phys. A 324, 487.

[2] Heger, A., Woosley, S. E., Martínez-Pinedo, G., and Langanke, K. (2001) Astro Phys. J. 560, 307.

[3] Langanke, K. and Martínez-Pinedo, G. (2003) Rev. Mod. Phys. 75, 819.

[4] Hix, W., Messer, O. E. B., Mezzacappa, A., Liebendörfer, M. M., Sampaio, J., Langanke, K., Dean, D. J., and Martínez-Pinedo, G. (2003) Phys. Rev. Lett. 91, 201102.

[5] Cole, A. L., Anderson, T. S., Zegers, R. G. T., Austin, S. M., Brown, B. A., Valdez, L., Gupta, S., Hitt, G. W., and Fawwaz, O. (2012) Phys. Rev. C 86, 015809.

[6] Rae, W. D. M. http://knollhouse.org/default.aspx.

[7] Brown, B. A. and Rae, W. D. M. NuShellX@MSU, B. A. Brown, Department of Physics and Astronomy, National Superconductin Cyclotron Laboratory, Michigan State Univeristy, East Lansing, Michigan 48824, USA and W. D. M. Rae, Garsington, Oxfordshire, OX44, UK http://www.nscl.msu.edu/ brown/resources/resources.html.

[8] Honma, M., Otsuka, T., Brown, B. A., and Mizusaki, T. (2002) Phys. Rev. C 65, 061301(R).

[9] Honma, M., Otsuka, T., Brown, B. A., and Mizusaki, T. (2004) Phys. Rev. C 69, 034335.

[10] Honma, M., Otsuka, T., Brown, B. A., and Mizusaki, T. (2005) Eur. Phys. J. A 25, 499.

[11] Poves, A., Sánchez-Solano, J., Caurier, E., and Nowacki, F. (2001) Nucl. Phys. A694, 157.

[12] Martínez-Pinedo, G., Poves, A., Caurier, E., and Zuker, A. P. (1996) Phys. Rev. C 53, R2602-R2605.

[13] Möller, P. and Randrup, J. (1990) Nucl. Phys. A 514, 1.

[14] Möller, P., Nix, J. R., Myers, W. D., and Swiatecki, W. J. (1995) At. Data Nucl. Data Tables 59, 185.

[15] Becerril-Reyes, A. D., Gupta, S., Schatz, H., Kratz, K.-L., and Möller, P. (2006) PoS NIC-IX, 075 and S. Gupta, private communication.

[16] Fuller, G. M., Fowler, W. A., and Newman, M. J. (1980) Astrophys. J. Supp. Series 42, 447.

[17] Fuller, G. M., Fowler, W. A., and Newman, M. J. (1982) Astrophys. J. 252, 715.

[18] Fuller, G. M., Fowler, W. A., and Newman, M. J. (1982) Astrophys. J. Sup. Ser. 48, 279.

[19] Fuller, G. M., Fowler, W. A., and Newman, M. J. (1985) Astrophys. J. 293, 1.

[20] Cole, A. L., Akimune, H., Austin, S. M., Bazin, D., van denBerg, A. M., Berg, G. P. A., Brown, J., Daito, I., Fujita, Y., Fujiwara, M., Gupta, S., Hara, K., Harakeh, M. N., JÃd'necke, J., Kawabata, T., Nakamura, T., Roberts, D. A., Sherrill, B. M., Steiner, M., Ueno, H., , and Zegers, R. G. T. (2006) Phys. Rev. C 74, 034333.

[21] Juodagalvis, A., Langanke, K., Hix, W., Martínez-Pinedo, G., and Sampaio, J. M. (2010) Nucl. Phys. A 848, 454.

[22] Langanke, K. and Martínez-Pinedo, G. (2004) Nucl. Phys. A 731, 365. 